\author{
Claudia Florida Costea ${ }^{1}$, Andrei Cucu ${ }^{2 *}$, \\ Victor Vlad Costan ${ }^{3}$, Gabriela Florența \\ Dumitrescu ${ }^{4}$, Anca Sava ${ }^{5}$ and Dana \\ Mihaela Turliuc ${ }^{2,6}$ \\ ${ }^{1}$ Department of Ophthalmology, Gr. T. Popa \\ University of Medicine and Pharmacy, Iassy, \\ Romania \\ ${ }^{2}$ Neurosurgery Unit II, Prof. Dr. Nicolae Oblu \\ Emergency Clinical Hospital, Iassy, Romania \\ ${ }^{3}$ Department of Surgery, Gr.T. Popa, University of \\ Medicine and Pharmacy of Iassy, Romania \\ ${ }^{4}$ Department of Pathology, Prof. Dr. Nicolae Oblu, \\ Emergency Clinical Hospital, Iassy, Romania \\ ${ }^{5}$ Department of Anatomy, Grigore T.Popa, \\ University of Medicine and Pharmacy, Iassy, \\ Romania \\ ${ }^{6}$ Department of Neurosurgery, Gr.T. Popa, \\ University of Medicine and Pharmacy, Iassy, \\ Romania
}

Dates: Received: 19 October, 2015; Accepted: 07 November, 2015; Published: 10 November, 2015

*Corresponding author: Andrei Cucu, MD, Neurosurgery Unit II, "Prof. Dr. Nicolae Oblu" Emergency Clinical Hospital, lasi, Romania, Tel:0040748378468; E-mail: andreiucucu@yahoo.com

www.peertechz.com

Keywords: Fibrous dysplasia; Visual impairment; Sphenoid sinus mucocele

ISSN: 2455-1414

\author{
Case Report
}

\section{Visual Impairment in Orbitofrontal and Sphenoidal Fibrous Dysplasia Associated with Sphenoid Sinus Mucocele}

\begin{abstract}
The orbitofrontal fibrous dysplasia is a rare, non-malignant disease that involves the development and thickening of craniofacial bones, causing facial asymmetry, visual, neurological and dental complications. Decreased visual acuity in orbitofrontal and sphenoidal fibrous dysplasia appears as a result of compressive lesions on the optic nerve. The aim of this article is to present the ophthalmologic complications due to the association of two entities that both involved the optic nerve: orbitofrontal fibrous dysplasia associated with a large sphenoid sinus mucocele. We report the case of a 20-years old male patient, who was admitted to the Neurosurgery Unit II of "Prof. Dr. Nicolae Oblu" Emergency Clinical Hospital, lassy, Romania, for headache, sudden decrease in visual acuity of both eyes in the last month, though more markedly in his right eye and facial deformity with right frontal bony bulging. The patient hadn't in his previous medical history any dermatological or endocrinological diseases. The ophthalmologic examination revealed that the visual acuity of the right eye was hand movements and best corrected visual acuity of the left eye was 20/60. Conventional X-ray, CT and MRI investigations showed an orbitofrontal and sphenoidal fibrous dysplasia associated with a large sphenoid sinus mucocele, located in the anterior and middle cranial fossa. Two surgical procedures were performed, the first being an attempt to perform a bilateral decompression of the optic nerve and in the second one, was evacuated the large sphenoid sinus mucocele, that compressed the optic chiasm. The first histopathological examination confirmed that the orbitofrontal and sphenoidal lesion had a typical appearance of fibrous dysplasia and the second biopsy revealed a sphenoid sinus mucocele. Six months after the evacuation of the sphenoid sinus mucocele, patient recovered completely his vision only in the left eye $(20 / 20)$ and the right eye had no light perception, he also developed a peripheral right facial nerve palsy.
\end{abstract}

\section{Introduction}

Fibrous dysplasia is an abnormal development of a bone mass in terms of structure and growth [1].

This is a rare disease with a benign evolution caused by the postzygotic activating mutations of the GNAS gene [2] on chromosome $20 \mathrm{q} 13$ [3] found in $71.9-86 \%$ of fibrous dysplasia cases [4] and that results in the inhibition of the differentiation and proliferation of the bone-forming stromal cells that leads to the replacement of the bone with fibrous tissue and immature thin bone trabeculae. Visual complications occur most often in case of spheno-ethmoid complex [5].

On the other hand, sphenoid sinus mucocele appears due to obstruction of mucinous gland ostium and can affect adjacent optic nerve, leading to visual impairment and even to blindness [6]. The aim of this article is to present the ophthalmologic complications due to the association of two entities that both involved the optic nerve: orbitofrontal fibrous dysplasia associated with a large sphenoid sinus mucocele.

\section{Case Presentation}

We report the case of a 20 -years old male patient, who was admitted to the Neurosurgery Unit II of "Prof.Dr.Nicolae Oblu" Emergency Clinical Hospital, Iassy, Romania for headache, sudden decrease in visual acuity of both eyes in the last month, though more markedly in his right eye and facial deformity. In his past medical history there was a progressive visual impairment, in the right eye for the last three years. The patient had no other significant diseases, nor any skin lesions, either precocious puberty or other endocrine abnormalities. General clinical examination revealed moderate facial deformity, with right frontal bony bulging.

The ophthalmological examination revealed a visual acuity of the right eye $(\mathrm{VA} \mathrm{RE})=$ hand movements and best corrected visual acuity of the left eye (VA LE) $=20 / 60$; there was an afferent pupillary defect in the right eye; the ophthalmoscopic examination revealed optic disc atrophy in the right eye and normally looking optic disc in the left eye.

Skull X-ray showed a "ground-glass" appearance of the frontal bone (Figure 1A).

Cranio-cerebral computed-tomography (CT) scan showed expansile, hyperdense and inhomogenous lesions, involving the frontal, ethmoid and sphenoid bones on both sides, yet predominantly on the right (Figure 1B), and a cystic and osteocondensed lesion located in the anterior and middle cranial fossa. 
The MRI scan of the head identified a well-defined neoformation, having 57/56/41 mm (ap / transverse / cranio-caudal), relatively homogeneous, with polycyclic contour, located in the sella turcica and parasellar,that extended predominantly anteriorly to the right ethmoid bone, but also posteriorly to the retrosellar region with compressive effect on the optic chiasm and neighboring structures (Figure 1C). Another extensive lesion affecting the right frontal bone, showed in homogeneous structure. The thickness of the right frontal bone had a diameter of 28/16 mm (Figure 1D).

There was also an incomplete development of the cerebral frontal lobes, especially of the right lobe, but no other significant pathological changes of the brain parenchyma. Bilateral hyperostosis of the great sphenoid wings could be seen. Right polypoid chronic maxillary sinusitis associated with moderate inflammatory features of its inner wall and a mild chronic ethmoiditis, predominantly on the right side (Figure 1B).

Based on these radiological findings a diagnosis of monostotic craniofacial dysplasia associated with a sphenoid sinus mucocele, causing bilateral partial obliteration of both optic canals with compression of both optic nerves was made.

The patient underwent two successive surgeries. The first was an attempt to perform bilateral decompression of the optic nerves and the second one aimed to evacuate the large sphenoid sinus mucocele, using a transfacial and transmaxillary approach with sampling of a sphenoid bone fragment for the histopathological exam (Figure 2).The first microscopical exam established the diagnosis of fibrous dysplasia, as the normal bone and marrow were replaced by fibrous tissue and haphazardly distributed thin bone trabeculae, seen as "Chinese letters" on the fibrous background (Figure 3, 4). The second lesion was a sphenoid sinus mucocele. Two weeks postoperatively, the patient had best corrected visual acuity of the left eye $20 / 20$, but there
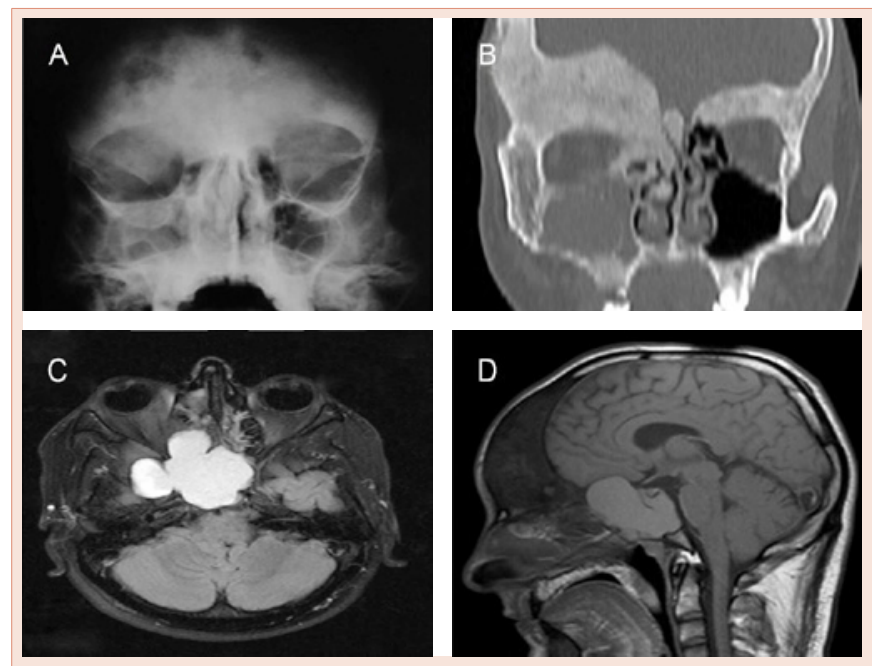

Figure 1: Orbitofrontal and sphenoidal fibrous dysplasia: A). Radiographic appearance - characteristic aspects of "ground glass"; B). CT scan appearance; C) MRI scan appearance: homogeneous well defined cystic lesion (57/56/41 $\mathrm{mm}$ ) located in the sellar, suprasellar and parasellar areas, predominantly on the right side, extending in the anterior cranial fossa, with compressive effect on the optic chiasm; D). T1-weighted MRI scan showing the large cyst and frontal dysplasia.

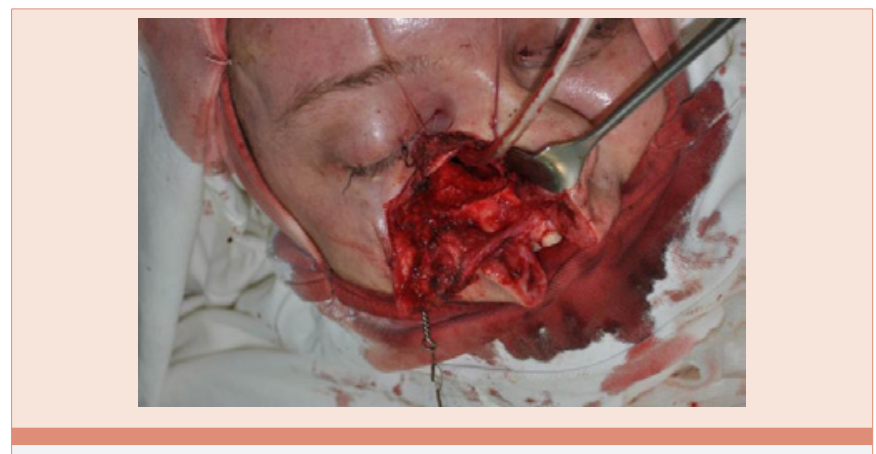

Figure 2: Intraoperative view-transmaxillary approach in our case of orbitofrontal and sphenoidal fibrous dysplasia with a large sphenoid sinus mucocele.

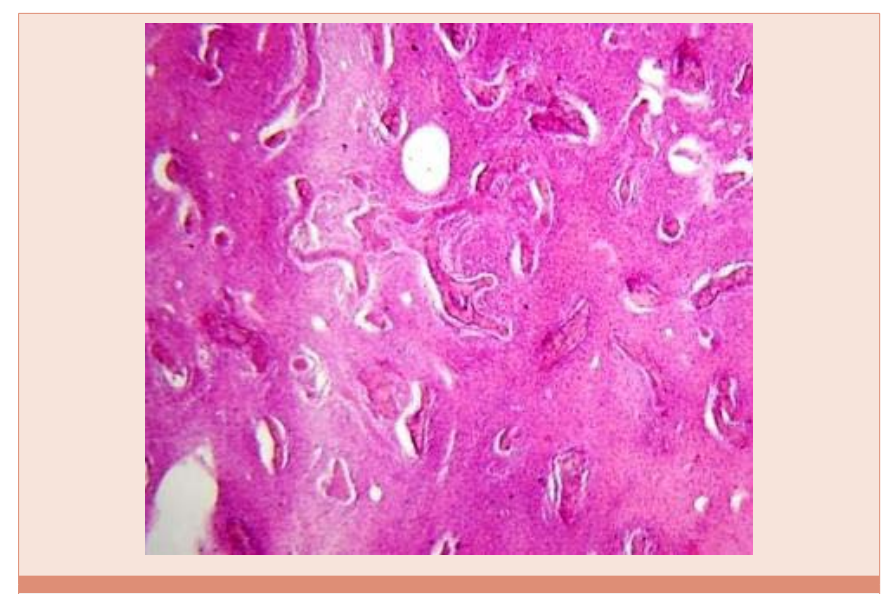

Figure 3: Fibrous dysplasia: normal bone was replaced with newly formed bone trabeculae, thin, irregular-looking like "Chinese letters", separated by fibrous connective tissue (H-E, x 40).

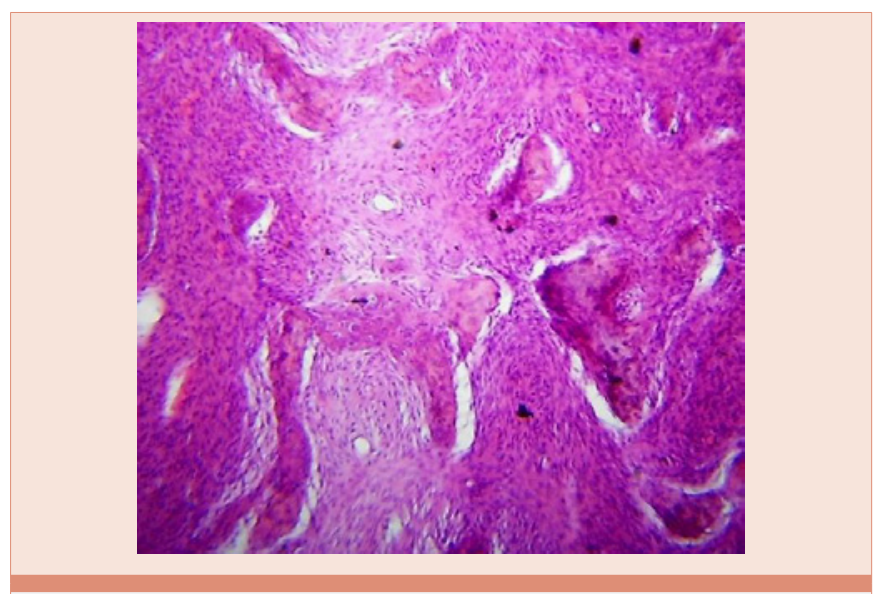

Figure 4: Microscopic field with lens of higher power highlights immature bone blades, poorly calcified bone pattern and with the presence of osteoblasts only from place to place. Form of the newly formed bone blades ranging from small oval islands, curves or serpiginouse, which gives the impression of "Chinese letters" or "alphabet soup" (H-E, x100).

was no light perception in the right eye. Later, the patient developed right-sided peripheral facial nerve palsy. Six months after the surgery, the ophthalmological examination revealed: VA LE $=20 / 20$ and VA $\mathrm{RE}=$ no light perception (NLP). The ophthalmoscopic examination 
revealed normally looking left optic disc and total secondary right optic disc atrophy.

\section{Discussions}

Fibrous dysplasia is a developmental disorder of growing bones [5], and its frequency varies between 5 - 7\% of all benign bone tumors [7]. The presentation forms of fibrous dysplasia are: the monostotic form (usually asymptomatic and incidental findings), polyostotic form as well McCune-Albright syndrome (polyostotic fibrous dysplasia, multiple endocrine abnormalities, skin pigmentation, precocious puberty) [8], and Mazabraud syndrome (intramuscular myxoma).

Craniofacial involvement in monostotic fibrous dysplasia could be found in only $10 \%-30 \%$, but the percentage is higher (50-100\%) in the polyostotic form $[5,9,10]$.

Among the craniofacial localizations of fibrous dysplasia, the maxillozygomatic complex is most frequent affected [11]. Ethmoid, sphenoid, temporal and frontal bones are rarely involved [12].

In cases of orbitofrontal sphenoidal fibrous dysplasia, the decrease of visual acuity is progressive, produced by bone proliferation and compression of the optic nerve (50-90\% of the cases) [13].

Sphenoid mucocele is also a rare condition comprising $1-2 \%$ of all mucoceles [14] with less than 150 cases reported until now. Mucocele is defined as the accumulation and retention of mucoid secretion within a paranasal sinus, leading to thinning and distension and erosion of one or several of its bony walls. Several hypotheses are postulated for the formation of mucocele, like blockage of duct, cystic dilation of mucous gland and cystic degeneration of polyp [15]. Sphenoidal mucocele have various clinical presentations because of adjacent non-bony structures, namely first six cranial nerves, the carotid arteries, the cavernous sinuses and the pituitary gland. Headache is the most common symptom [16]. Visual impairment is the second-most common symptom, and is mostly because of optic nerve involvement. It can lead to decreased visual acuity and even blindness, which is usually irreversible [17]. Visual disturbance can occur because of the third nerve involvement [18].

Investigations for diagnosing sphenoid mucocele include plain X-ray of skull A.P. and Lat. views, which can show enlargement and ballooning of the sella with erosion of its walls. CT scan of the brain in case of sphenoid mucocele would show a hypodense cystic lesion in the sphenoid sinus with or without extension to the adjacent sellar, suprasellar, parasellar and retrosellar regions. It has to be differentiated with other cystic lesions usually seen in this location, such as craniopharygioma, Rathke cleft cyst, cystic pituitary adenoma, epidermoid cyst, cystic optic nerve glioma and arachnoid cyst. MRI scans of the brain can diagnose mucocele without doubt as a cystic homogenous lesion in relation to the paranasal sinuses [14,19]. These lesions tend to extend and expand along the path of least resistance. The slow and silent expansion of a mucocele may be unsuspected until bone is eroded and it impinges on adjacent structures.Sphenoidal mucoceles generally tend to spread more frequently in an anteriorinferior fashion with invasion of the ethmoid, the nasal fossae and the nasopharynx. It may show upward invasion with destruction of the sellar floor. There may be an invasion in the orbital cavity when spreading occurs sideways. More rarely, the intermediate cranial fossa is invaded through the lateral wall and the posterior cranial fossa through the posterior wall [20].

In our case, visual impairment in both eyes was initially determined by the progressive compression of the optic canals, predominantly of the right side, due to the orbitofrontal fibrous dysplasia. Sudden visual impairment, more markedly in the patient's right eye appeared due to supplementary compression of the optic chiasm and right optic nerve by the large sphenoid sinus mucocele; after the evacuation of the mucocele, visual acuity fully recovered in the patient's left eye (20/20). The patient's visual acuity of the right eye remained without light perception due to right secondary optic disc atrophy.

Patients diagnosed with craniofacial fibrous dysplasia often involves facial asymmetry, exophthalmia, limitation of eye movements and decreased visual acuity [21], due to compression of the optic nerve [22]. Impaired vision is the most feared complication [21], and is the most common complication of fibrous dysplasia that affects the craniofacial bones [23], especially the sfeno-ethmoid complex [5]. In craniofacial fibrous dysplasia there are some other ocular manifestations that could be associated with decreased visual acuity, such as impaired sense of color, visual field defects and pupillary defect. All of them would appear in a progressive or acute manner [13,21].

The compressive effect of craniofacial fibrous dysplasia on the optic nerve and optic chiasm can be identified using CT and MRI scans.

From radiographic point of view, there are three forms of fibrous dysplasia [24]: the compact form (50\% of the cases), the lytic form and the mixed form. Our patient had the compact form of fibrous dysplasia which affected the sphenoid bone, and bilateral orbital roofs and frontal bones.

The MRI aspect depends on the degree of fibrous tissue cellularity and the number of bone trabeculae [25]. The differential diagnosis may be carried out by: chordoma, chondroma, hyperstotic meningioma, osteoma, osteosarcoma, hyperostosis and ossified fibroma. Surgery is indicated in symptomatic cases. In asymptomatic cases, in which fibrous dysplasia impairs important structures, clinical monitoring and repeated imaging are recommended [26].

Conservative treatment may be indicated in patients with unaffected visual acuity accompanied by radiological control, interpreted in the context of clinical correlations [21]. Treatment with corticosteroids has been used successfully in patients with fibrous dysplasia as a temporary measure in patients who have loss of visual acuity [27], but it has not been used in patients complaining of recurrent of visual acuity [21]. Surgical treatment is controversial and consists of preventive or therapeutic decompression of optical channel [1,28-30], which leads many times to loss of visual acuity in the affected eye or to an increased incidence of visual acuity changes $[23,28]$.

In our patient, the first surgery was an attempt to decompress the bilateral optic nerve and the second one to evacuate the large sphenoid sinus mucocele, in order to decompress the optic chiasm. 
Postoperatively, the patient had fully recovered sight in his left eye, but his right eye sight was permanently impaired, the right optic nerve being already atrophied.

In orbitofrontal fibrous dysplasia, the surgical approach is set out depending on the location of the lesion. Most lesions may be reached by anterior approach: transfacially, through the coronary approach or endoscopically [31]. The lesions which are limited on the frontal, ethmoidal or sphenoidal region may be respected by an endonasal approach. In our case, the surgical approach was performed through the right maxilla.

\section{Conclusion}

In the case reported here, the patient was diagnosed late, when he was admitted to the Neurosurgery Unit, for bilateral visual acuity impairment, headache and facial deformity. Optic nerve and optic chiasm compression was due to the association of two entities that both involved the optic nerve: orbitofrontal fibrous dysplasia associated with a large sphenoid sinus mucocele, which caused right optic nerve atrophy and permanent visual impairment in the right eye.

The patient should see the physician at first clinical signs, as to make the treatment possible at an appropriate time, so as he should not be left with serious sequelae, particularly the permanent impairment of the visual acuity.

\section{Conflict of Interests}

The authors declare that they have no conflict of interests.

\section{References}

1. Abe T, Sato K, Otsuka T, Kawamura N, Shimazu M, et al. (2002) Optic Nerve decompression for orbitofrontal fibrous dysplasia. Skull Base: An Interdisciplinary Approch 12: 145-152.

2. Schwindinger WF, Francomano CA, Levine MA (1992) Identification of a mutation in the gene encoding the alpha subunit of the stimulatory $G$ protein of adenylyl cyclase in McCune-Albright syndrome. Proc Natl Acad Sci USA 89: $5152-5156$

3. Weinstein LS, Chen M, Liu J (2002) Gs(alpha) mutations and imprinting defects in human disease. Ann NY Acad Sci 968: 173-197.

4. Tabareau-Delalande F, Collin C, Gomez-Brouchet A, Decouvelaere AV Bouvier C, et al. (2013) Diagnostic value of investigating GNAS mutations in fibro-osseous lesions: a retrospective study of 91 cases of fibrous dysplasia and 40 other fibro-osseous lesions. Mod Pathol 26: 911-921.

5. Alam A, Chander BN (2003) Craniofacial Fibrous Dysplasia presenting with Visual Impairment. MJAFI 59: 342-343.

6. Sharifi G, Jalessi M, Erfanian D, Farhadi M (2013) Sudden blindness due to isolated sphenoid sinus mucocele and retention cyst. Braz J Otorhinolaryngol 79: 120.

7. MacDonald-Jankowski D (2009) Fibrous dysplasia: a systematic review. Dentomaxillofac Radiol 38: 196-215.

8. Albright FBA, Hampton AO, Smith P (1937) Syndrome characterized by osteitis fibrosa disseminata, areas of pigmentation and endocrine dysfunction, with precocious puberty in females: report of five cases. N Engl J Med 216: 727-746

9. Hudson TM, Stiles RG, Monson DK (1993) Fibrous lesions of bone. Radio
Clin North Am 31: 279-297.

10. Eversole LR, Sabes WR, Rovin S (1972) Fibrous dysplasia: a nosologic problem in the diagnosis of fibro-osseous lesions of the jaws. J Oral Pathol 1: 189-220.

11. Lee JS, FitzGibbon E, Butman JA, Dufresne CR, Kushner H, et al. (2002) Normal vision despite narrowing of the optic canal in fibrous dysplasia. $\mathrm{N} \mathrm{Engl}$ J Med 347: 1670-1676.

12. Mohammadi-Araghi H, Haery C (1993), Fibrous-osseous lesions of craniofacial bones. The role of imaging.Radiol Clin North Am 31(1):121-134.

13. Katz BJ, Nerad JA (1998) Ophthalmic manifestations of fibrous dysplasia: a disease of children and adults. Ophthalmology 105: 2207-2215.

14. Kataria R, Gupta S, Chopra S, Bagaria H, Sinha VD (2012) Mucocele of the sphenoid sinus: A rare cause of reversible $3^{\text {rd }}$ nerve palsy. Ann Indian Acad Neurol 15: 158-160.

15. Friedman A, Batra PS, Fakhri S, Citardi MJ, Lanza DC (2005) Isolated sphenoid sinus disease: Etiology and management. Otolaryngol Head Neck Surg 133: $544-550$

16. Nugent GR, Sprinkle P, Bloor BM (1970) Sphenoid sinus mucoceles. J Neurosurg 32: 443-451.

17. Casteels I, De Loof E, Brock P, Jorissen M, Dralands L, et al. (1992) Sudden blindness in a child: Presenting symptom of a sphenoid sinus mucocele. $\mathrm{Br} \mathrm{J}$ Ophthalmol 76: 502-504

18. Friedmann G, Harrison S (1970) Mucocoele of the sphenoidal sinus as a cause of recurrent oculomotor nerve palsy. J Neurol Neurosurg Psychiatry 33:172-179

19. Akan H, Cihan B, Çelenk Ç (2004) Sphenoid sinus mucocele causing third nerve paralysis: CT and MR findings. Dentomaxillofacial Radiology 33: 342344.

20. Cerqua N, D'Ottavi LR, Coen-Tirelli G, Piccirillo E (1996) Mucocele of sphenoid sinus: presentation of 3 cases. Acta Otorhinolaryngol Ital 16: 52-56.

21. Singh T, Singh S, Vyas K (2015) Presentation of Fibrous Dysplasia. Internet Scientific Publications, on-line.

22. Michael CB, Lee AG, Patrinely JR, Stal S, Blacklock JB (2000), Visual loss associated with fibrous dysplasia of the anterior skull base.Case report and review of the literature. J Neurosurg 92(2):350-354.

23. Sassin JF, Rosenberg RN (1968) Neurological complications of fibrous dysplasia of the skull. Arch Neurol 18: 363-369.

24. Derome PJ, Visot A, Akerman M, Oddou B, Mazabraud A, et al. (1983) La dysplasie fibreuse crânienne. Neurochirurgie 29: 1-117.

25. MacDonald-Jankowski DS (2004) Fibro-osseous lesions of the face and jaws. Clin Radiol 59: 11-25.

26. Cutler CM, Lee JS, Butman JA, FitzGibbon EJ, Kelly MH, et al. (2006) Longterm outcome of optic nerve encasement and optic nerve decompression in patients with fibrous dysplasia: risk factors for blindness and safety of observation. Neurosurgery 59: 1011-1017.

27. Arroyo JG, Lessell S, Montgomery WW (1991) Steroid-induced visual recovery in fibrous dysplasia. J Clin Neuroopthalmol 11: 259-261.

28. Chen YR, Breidahl A, Chang CN (1997) Optic nerve decompression in fibrous dysplasia: indications, efficacy and safety. Plast Reconstr Surg 99: 22-30.

29. Edgerton MT, Persing JA, Jane JA (1985) The surgical treatmewnt of fibrous dysplasia with emphasia with wmphasis on recent contributions from craniomaxillo-facial surgery. Ann Surg 202: 459-479.

30. Papay FA,Morales L Jr, Flaharty P, Smith SJ, Anderson R, WAlker JM, Hood RS, Hardy S (1995), Optic nerve decompression in cranial base fibrous dysplasia. J Craniofac Surg 6(1):5-10.

31. Ikeda K, Suzuki H, Oshima T, Shimomura A, Nakabayashi S, et al. (1997), Endonasal endoscopic management in fibrous dysplasia of the paranasal sinuses. Am J Otolaryngol 18: 415-418.

Copyright: (c) 2015 Costea CF, et al. This is an open-access article distributed under the terms of the Creative Commons Attribution License, which permits unrestricted use, distribution, and reproduction in any medium, provided the original author and source are credited. 